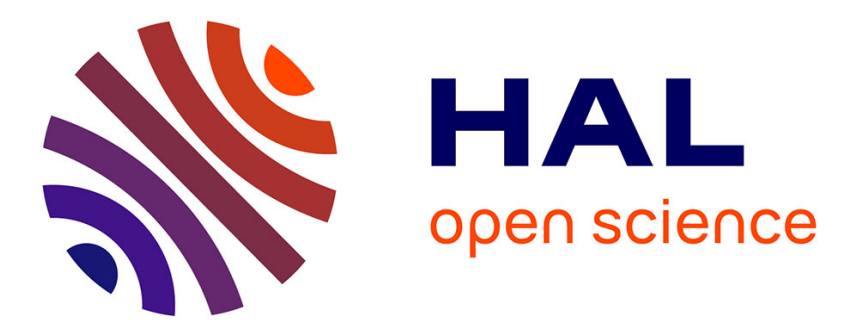

\title{
Les sources scientifiques de Jules Bourgoin : cristaux, polygones et polyèdres
}

\author{
Jenny Boucard, Christophe Eckes
}

\section{To cite this version:}

Jenny Boucard, Christophe Eckes. Les sources scientifiques de Jules Bourgoin : cristaux, polygones et polyèdres. Bideault, M. \& Thibault, E. \& Volait, M. De l'Orient à la mathématique de l'ornement: Jules Bourgoin (1838-1908), Picard, pp.299 - 318, 2015, 10.4000/books.inha.7037 . halshs-01351717

\section{HAL Id: halshs-01351717 https://shs.hal.science/halshs-01351717}

Submitted on 14 Apr 2021

HAL is a multi-disciplinary open access archive for the deposit and dissemination of scientific research documents, whether they are published or not. The documents may come from teaching and research institutions in France or abroad, or from public or private research centers.
L'archive ouverte pluridisciplinaire HAL, est destinée au dépôt et à la diffusion de documents scientifiques de niveau recherche, publiés ou non, émanant des établissements d'enseignement et de recherche français ou étrangers, des laboratoires publics ou privés. 
De l'Orient à la mathématique de l'ornement. Jules

Bourgoin (1838-1908)

Publications de l'Institut national d'histoire de l'art

\section{Les sources scientifiques de Jules Bourgoin : cristaux, polygones et polyèdres}

Jenny Boucard et Christophe Eckes

DOI : 10.4000/books.inha.7037

Éditeur : Publications de l'Institut national d'histoire de l'art, Picard

Lieu d'édition : Paris

Année d'édition : 2015

Date de mise en ligne : 5 décembre 2017

Collection : InVisu

ISBN électronique : 9782917902813

\section{Oanartition ebooks}

http://books.openedition.org

Édition imprimée

Date de publication : 1 octobre 2015

Ce document vous est offert par Université de Nantes

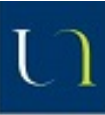

UNIVERSITÉ DE NANTES

\section{Référence électronique}

BOUCARD, Jenny; ECKES, Christophe. Les sources scientifiques de Jules Bourgoin : cristaux, polygones et polyèdres In : De l'Orient à la mathématique de l'ornement. Jules Bourgoin (1838-1908) [en ligne]. Paris Publications de l'Institut national d'histoire de l'art, 2015 (généré le 10 avril 2021). Disponible sur Internet : <http://books.openedition.org/inha/7037>. ISBN : 9782917902813. DOI : https://doi.org/ 10.4000/books.inha.7037

Ce document a été généré automatiquement le 10 avril 2021. 


\title{
Les sources scientifiques de Jules Bourgoin : cristaux, polygones et polyèdres
}

\author{
Jenny Boucard et Christophe Eckes
}

1 L'œuvre de Jules Bourgoin atteste de son intérêt constant pour les sciences mathématiques, la cristallographie, la chimie et la botanique. Il vaut donc la peine de s'interroger sur la nature, le statut et les usages de ses sources scientifiques en les reliant à ses travaux dans le domaine de l'ornement. L'autorité du philosophe et mathématicien Antoine-Augustin Cournot conditionne en partie le corpus d'ouvrages scientifiques consultés par Bourgoin, en particulier à partir de l'intérêt que Cournot accorde à la combinatoire - ou, selon ses propres termes, à la «syntactique » - et à la théorie de l'ordre ${ }^{1}$. Cependant, l'examen des notes de lecture conservées dans les différents fonds d'archives ${ }^{2}$ de Bourgoin montre que ses sources scientifiques excèdent largement cette référence première à Cournot. Une approche globale de ses notes révèle à la fois l'étendue et la grande cohérence de ses lectures, centrées sur quelques thématiques bien choisies.

2 L'importance de ce corpus scientifique est indéniable. Mais ce constat se heurte à un paradoxe : dans tous ses ouvrages, Bourgoin refuse d'annexer les arts d'ornement aux sciences. Quel usage fait-il alors de ses sources scientifiques? Un premier panorama permet de les thématiser et d'en préciser les emplois. Ainsi, l'exemple des cristaux de neige montre comment l'étude d'ouvrages scientifiques sert son projet d'analyse des formes ornementales; quant à la thématique des polygones et des polyèdres, elle souligne la richesse de son corpus plus spécifiquement mathématique sur un même thème et la variété de ses usages.

\section{Un panorama des sources scientifiques de Bourgoin}

Quelles sont les conditions qui ont permis à Bourgoin d'accéder à des sources scientifiques nombreuses et variées? Durant sa formation en architecture à la fin des 
années 1850 à l'École des beaux-arts, Bourgoin suit des cours de géométrie et de statique dispensés par Isidore Francœur, fils du mathématicien Louis-Benjamin Francœur qui est notamment connu pour ses manuels de mathématiques et de dessin linéaire. Les carnets de notes de Bourgoin ${ }^{3}$ indiquent, de manière plus surprenante, qu'il a également suivi des cours en arithmétique dont le niveau avancé excède les programmes enseignés à l'École des beaux-arts, peut-être ceux qu'Isidore Francœur dispense au collège Chaptal. Les connaissances initiales de Bourgoin vont donc au-delà de sa formation d'architecte et lui permettent par exemple de lire assez tôt des ouvrages exigeant des compétences tant en arithmétique, en algèbre qu'en géométrie. C'est par exemple le cas du livre de Cournot sur la correspondance entre l'algèbre et la géométrie que Bourgoin étudie de manière approfondie ${ }^{4}$.

Plusieurs hypothèses peuvent être émises sur ses moyens d'accès aux sources. Dans ses jeunes années, il a pu construire une partie de son corpus à partir des ouvrages de mathématiques - plus spécifiquement, de géométrie élémentaire, de géométrie descriptive et de perspective linéaire - disponibles à la bibliothèque de l'École des beaux-arts ${ }^{5}$. Bourgoin s'est ensuite constitué au fil de ses lectures des listes bibliographiques qui l'ont conduit à effectuer des recherches plus précises, notamment à la Bibliothèque nationale et à la bibliothèque Sainte-Geneviève. Par ailleurs, son inventaire après décès ${ }^{6}$ indique qu'il possédait divers ouvrages scientifiques et philosophiques, mais n'en détaille pas les titres.

5 Les listes bibliographiques établies par Bourgoin sont de plusieurs types : elles peuvent être centrées autour d'une thématique - comme les polyèdres, les figures de la neige ou encore les végétations métalliques - ou d'un auteur. Bourgoin semble aussi avoir eu pour habitude d'effectuer des recherches sur des périodiques scientifiques, académiques ou non ${ }^{7}$, soit ponctuelles, soit pour y repérer des thématiques prédéfinies ou pour sélectionner certaines planches d'illustrations. Pour constituer ses listes de références, Bourgoin a pu s'appuyer sur des notices biographiques - c'est par exemple le cas de la biographie de Gaspard Monge par Charles François Maximilien Marie de Ficquelmont - ou encore sur des manuels, des traités et des ouvrages à caractère généraliste, encyclopédique ou historique sur les mathématiques et les sciences.

6 Ainsi Bourgoin a construit en érudit et méthodiquement son corpus de textes scientifiques. Encore faut-il déterminer clairement l'objet de ses lectures scientifiques ainsi que leurs usages en les reliant à ses ouvrages.

7 La formation initiale de Bourgoin en architecture a sans doute une incidence sur le type de publications scientifiques qu'il consulte. Mais si la géométrie pure et la géométrie appliquée à l'architecture ainsi qu'à divers secteurs industriels représentent les domaines des mathématiques les plus exploités par Bourgoin, il s'est également intéressé à d'autres branches des mathématiques - combinatoire, arithmétique, algèbre, théorie des courbes et des surfaces courbes - et à d'autres domaines scientifiques : mécanique, cristallographie, stéréochimie, optique ou encore botanique.

8 En botanique, Bourgoin s'intéresse essentiellement à des études sur la forme des végétaux et la disposition de leurs parties. Il privilégie cette approche formelle puisque, de son côté, il traite les végétaux au même titre que les motifs ornementaux dont il cherche à dégager l'ossature géométrique, les rapports de situation, ainsi que les symétries. Pour ce faire, il s'inspire des travaux des frères Bravais et de plusieurs publications dues à Charles Fermond ${ }^{8}$ (fig. 1). Bourgoin consacre ainsi quelques pages aux «formes organiques» dans plusieurs de ses ouvrages ${ }^{9}$. Il se constitue également 
toute une gamme de formes renvoyant aux cristaux de neige. Plusieurs références bibliographiques et reproductions d'introductions d'ouvrages sont en lien avec les domaines de la stéréochimie et de la cristallographie, qui supposent une approche formelle et géométrique, fondée sur l'étude de la disposition des atomes au sein des molécules et des substances cristallines. Bourgoin étend également cette approche combinatoire aux sons et aux couleurs, ainsi qu'à leurs correspondances, étudiant notamment les travaux fondateurs de Michel-Eugène Chevreul ${ }^{10}$.

\section{J. Bourgoin, Notes d'après Ch. Fermond, Essai de phytomorphie, 1864.}

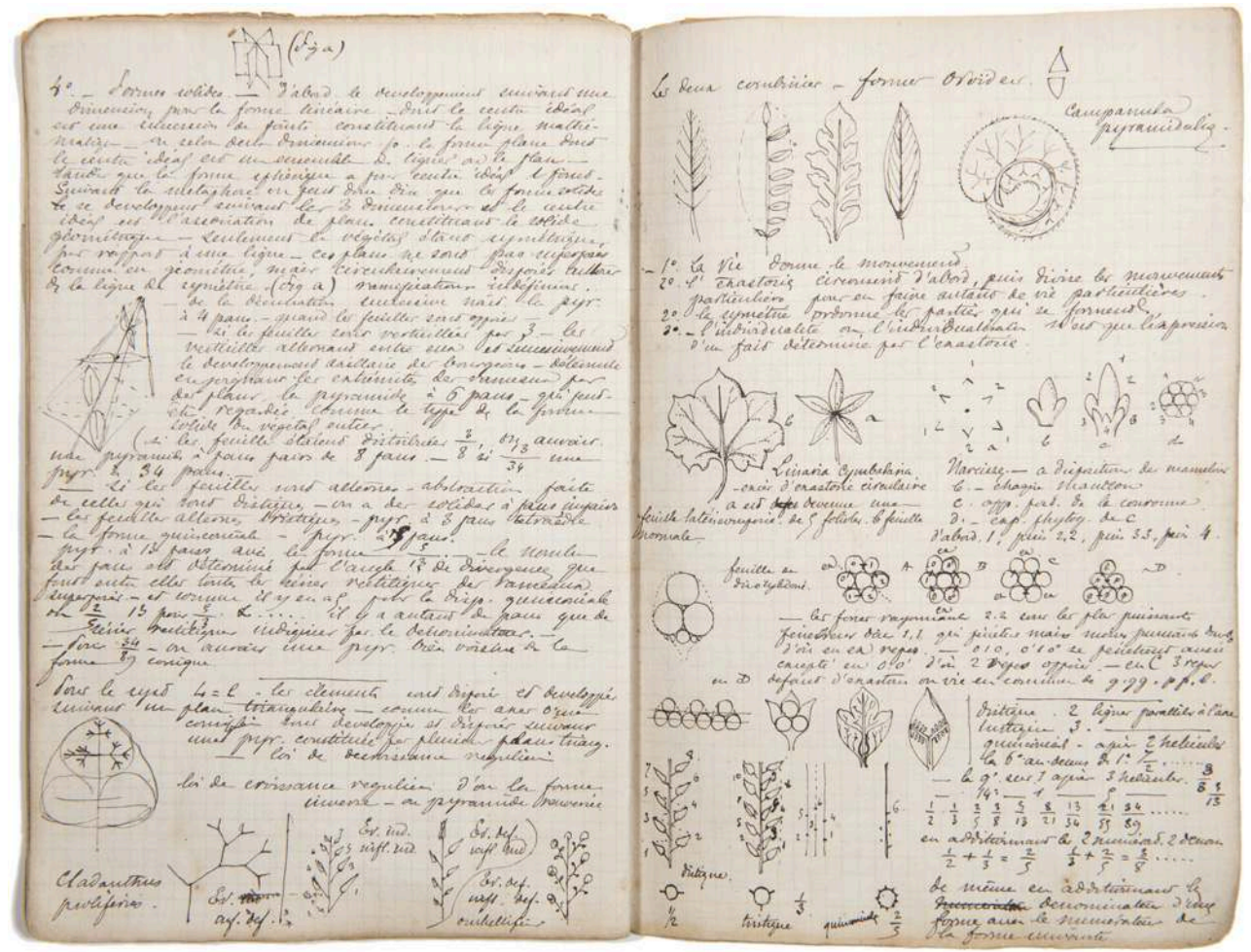

Source : Paris (France), Bibliothèque de l'Institut national d'histoire de l'art, collections Jacques Doucet, Arch. 67, 11, 4

Enfin, dans le cas des sciences mathématiques, les références de Bourgoin sont plus nombreuses et plus variées. Sont consultés divers manuels de géométrie élémentaire et descriptive, des ouvrages généralistes, historiques mais également des articles plus spécialisés tirés du Journal de l'École polytechnique ou des Comptes rendus de l'Académie des sciences notamment. Les notes de Bourgoin attestent d'une familiarité avec les sciences mathématiques, bien au-delà du simple recopiage (comme c'est le cas pour la stéréochimie). Il n'hésite pas à rassembler des citations issues de divers chapitres d'un même livre et il juxtapose parfois même sur une seule page des passages empruntés à différents ouvrages. Autrement dit, sa lecture de tels manuels n'est pas seulement linéaire, elle est également transversale, ce qui prouve qu'il maîtrise davantage ses sources. En outre, parmi les extraits de manuels de géométrie reproduits par Bourgoin, on trouve des démonstrations de théorèmes élémentaires et des résolutions de problèmes, figures à l'appui. Ces différentes données attestent d'une pratique effective et régulière des mathématiques chez l'auteur. 
10 Parmi ses notes figurent de nombreux emprunts à des manuels de géométrie élémentaire destinés à la préparation aux concours d'entrée aux écoles Normale supérieure et Polytechnique, ainsi que des références récurrentes à des manuels de géométrie descriptive visant un public d'ingénieurs et d'architectes. Ces manuels sont notamment utilisés pour la rédaction des Études architectoniques et graphiques, soit à la fin des années 1890. La forme des notes correspondantes, qui sont du type «brouillons d'exercices ", montre que le deuxième tome résulte d'une appropriation en profondeur des traités de géométrie élémentaire. Après ce bref panorama, nous nous proposons de préciser les usages que Bourgoin fait de ses sources scientifiques à partir de deux exemples : les cristaux de neige d'une part, les polygones et les polyèdres d'autre part.

\section{Les figures de la neige}

11 Deux planches dédiées aux cristaux de neige devaient figurer dans un projet inabouti de "Revue de l'ornement ${ }^{11}$ " (fig. 2 et 3). Cet exemple permet de montrer comment l'« ambition encyclopédiste ${ }^{12}$ » de Bourgoin guide sa manière de recenser et de choisir, parmi diverses sources scientifiques, les motifs des cristaux de neige qui l'intéressent.

\section{J. Bourgoin, «Étoiles de neige ».}

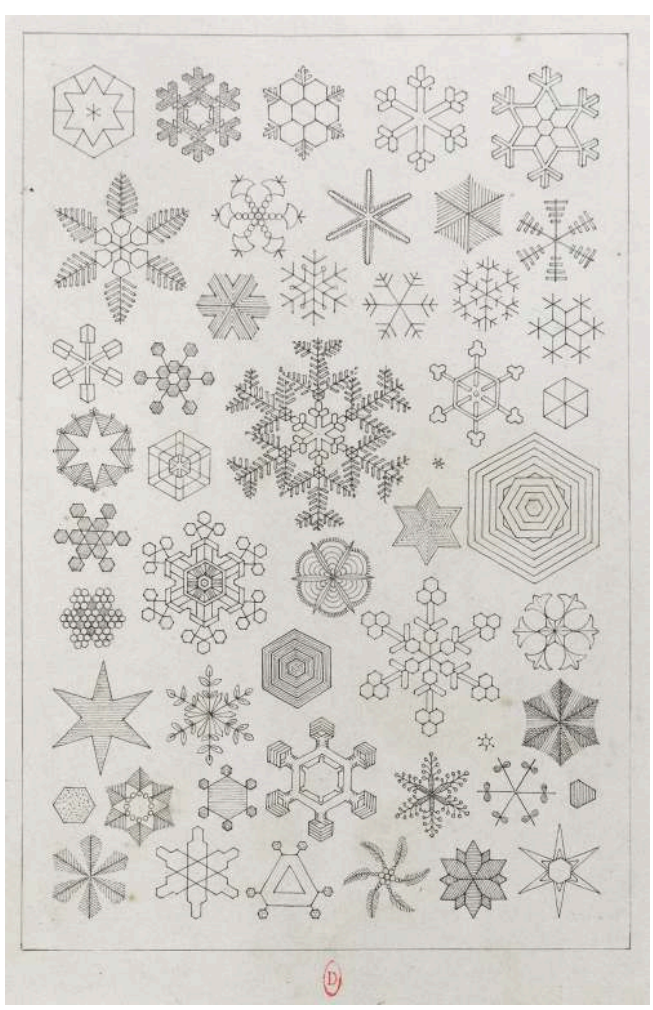

Source: Paris (France), Bibliothèque de l'Institut national d'histoire de l'art, collections Jacques Doucet, Arch. 67, 15, 7 


\section{J. Bourgoin, «Étoiles de neige ».}

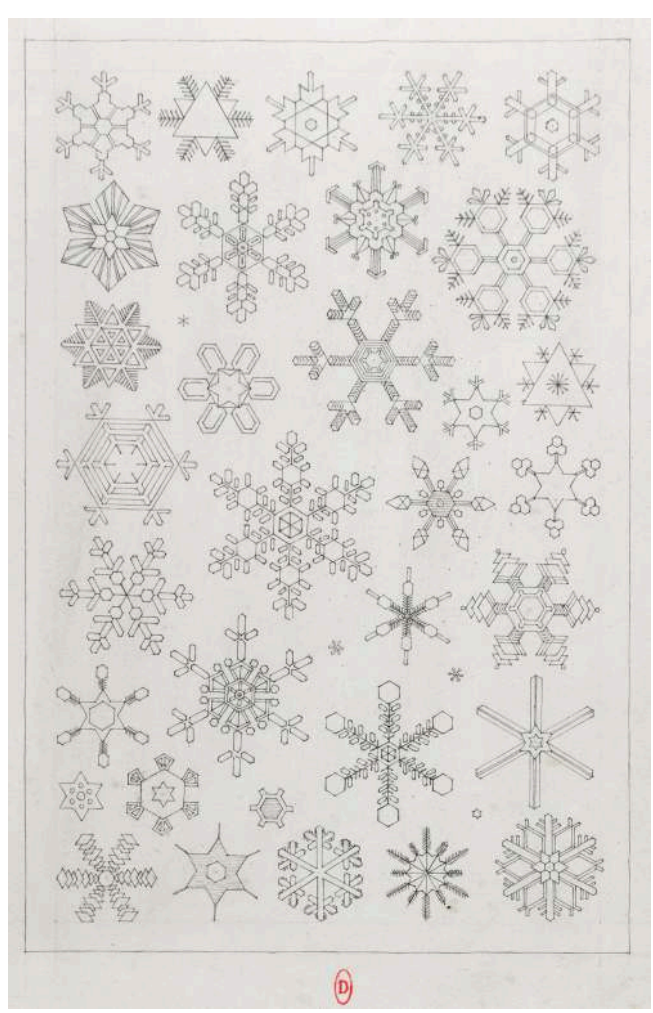

Source : Paris (France), Bibliothèque de l'Institut national d'histoire de l'art, collections Jacques Doucet, Arch. 67, 15, 7.

Plusieurs croquis préparatoires à ces deux planches, auxquels il faut ajouter diverses listes bibliographiques associées à la thématique des figures de la neige, documentent la démarche. Ce thème mobilise de nombreux scientifiques depuis le XvII siècle. Les sources consultées par Bourgoin parcourent toute cette période et il répertorie en bibliothèque l'ensemble des planches de cristaux de neige existantes. Pour ce faire, il s'appuie sur une source mère récente, à savoir l'ouvrage de Georg Friedrich Schumacher ${ }^{13}$ qui lui permet de remonter vers un large corpus. En effet, ce livre contient une notice historique détaillée dans laquelle sont référencés les principaux travaux sur les cristaux de neige depuis Kepler, Descartes et Hooke, pour aller jusqu'aux classifications exhaustives des types de cristaux de neige par William Scoresby au XIX ${ }^{e}$ siècle. Bourgoin a non seulement étudié ce livre de Schumacher, puisqu'il s'y réfère plusieurs fois dans ses notes et qu'il en a recopié toutes les planches, mais il en a aussi consulté le corpus de références.

Bourgoin commence par mentionner dans ses notes Descartes et il relève avec précision les références de la planche de la Micrographia de Hooke sur les cristaux de neige $^{14}$ (fig. 4). Il répertorie ensuite l'ensemble des écrits et planches de Pieter van Musschenbroek sur les figures de la neige ${ }^{15}$. Notons que ces trois savants sont évoqués dans la plupart des articles consacrés à l'histoire des découvertes sur le sujet ${ }^{16}$. 
4. R. Hooke, pl. VIII de Micrographia, 1667.

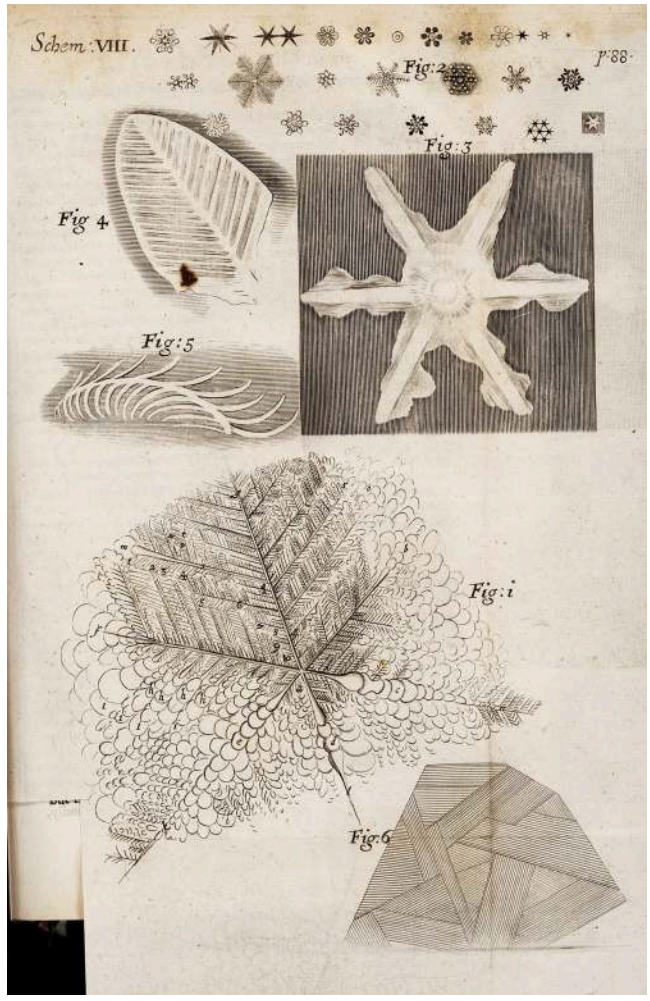

La lecture de Schumacher, qui fait référence à un article de Leonardus Stocke dans les Philosophical Transactions, met également Bourgoin sur la piste de plusieurs volumes de ce périodique. Passant en revue les années 1673 à 1761, il identifie des articles sur les cristaux de neige qui ne sont pas recensés par Schumacher. La mention «Dr Grew " barrée et visiblement mal orthographiée apparaît dans les notes de Bourgoin, elle correspond à un court texte du botaniste et médecin Nehemia Grew dans le volume de 1673 ; le nom du « Dr Langwith », pour Benjamin Langwith renvoie à un autre article ${ }^{17}$. Bourgoin a par ailleurs reproduit les quatre figures de cristaux de neige qui accompagnent l'article plus tardif de Stocke dans ce même périodique. Mais surtout, grâce à l'ouvrage de Schumacher, Bourgoin isole le nom de Johannes Nettis dont l'article en latin sur les cristaux de neige est traduit en anglais dans les Philosophical Transactions en 1756. Bourgoin recopie intégralement les deux planches recensant au total 91 formes distinctes de cristaux de neige, en en respectant scrupuleusement la numérotation $^{18}$ (fig. 5 et 6 ). 
5. J. Nettis, pl. XX du vol. 49 des Philosophical Transactions, 1756.

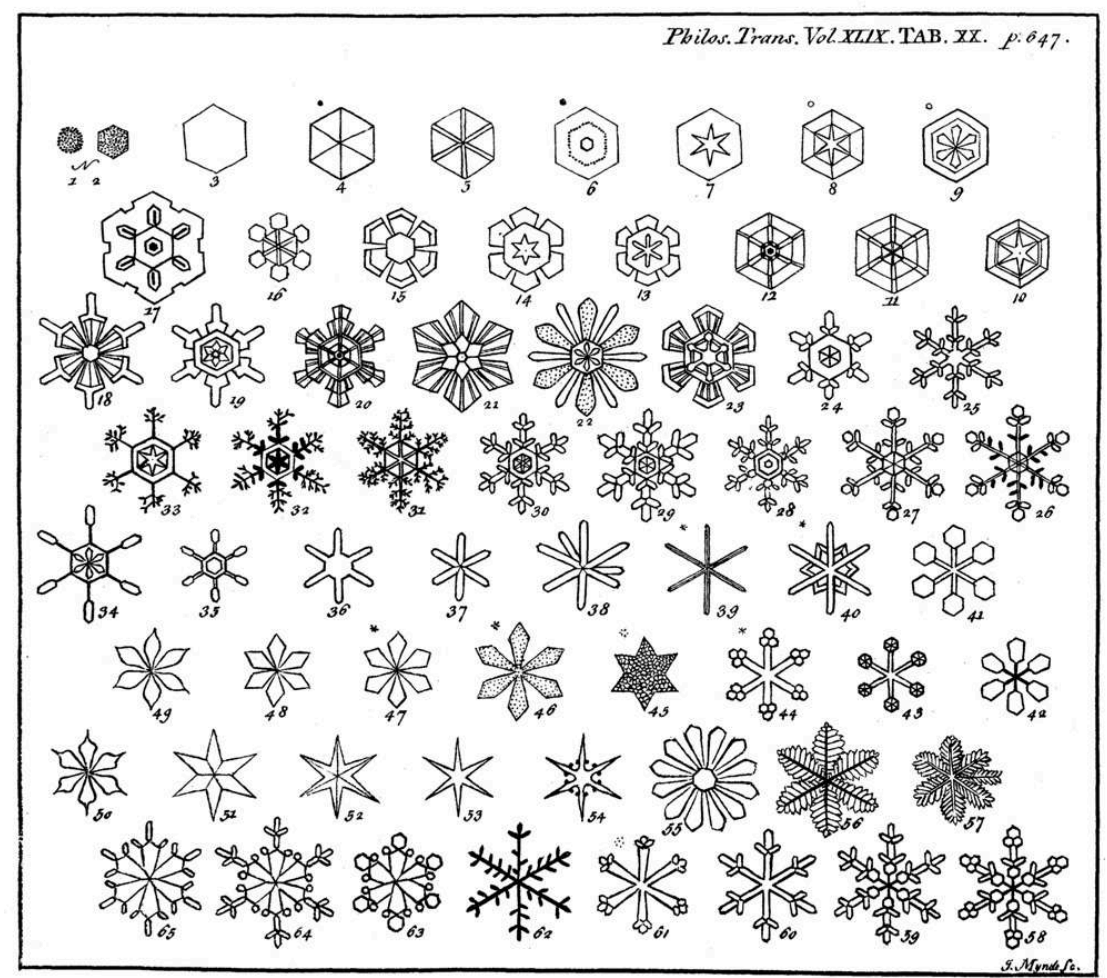

6. J. Bourgoin, Notes d'après J. Nettis.

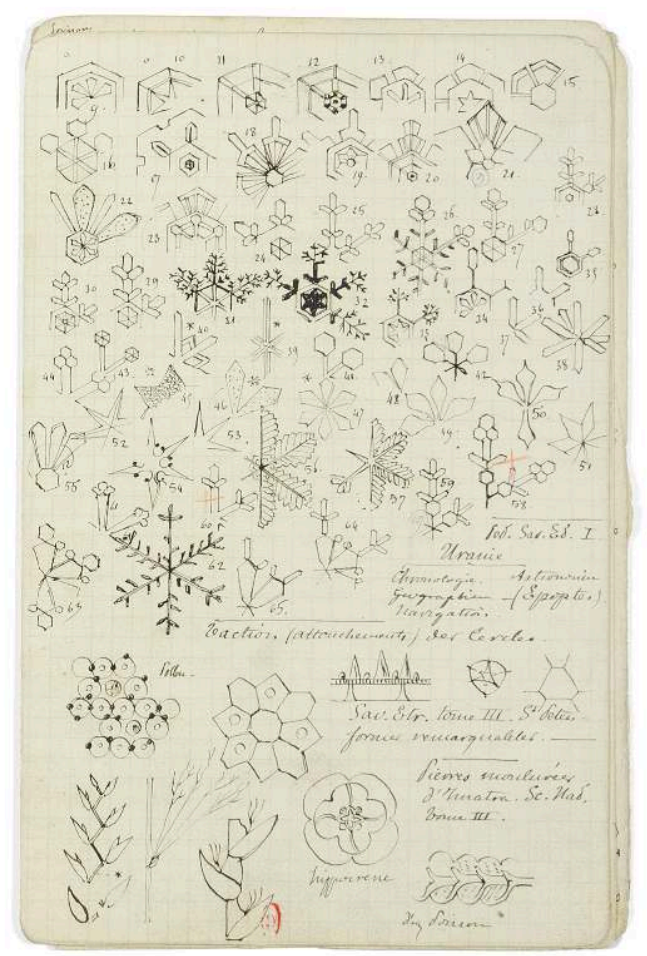

Source : Paris (France), Bibliothèque de l'Institut national d'histoire de l'art, collections Jacques Doucet, Arch. 67, 2, 1. 
15 Bourgoin, dont l'une comporte la mention: "Wilke (Actes de Suède pour 1760 et 1761)», suivie d'une référence en anglais et en allemand: "Swedish Academy, Abhandlungen tome XXIII ». Bourgoin recopie partiellement, à partir de l'ouvrage de Schumacher, les références d'un article de Johann Carl Wilcke ${ }^{19}$ sur les cristaux de neige.

D'ailleurs, Bourgoin complète sa gamme de figures de la neige en s'appuyant sur les ouvrages de Scoresby ${ }^{20}$ et de Schumacher (fig. 7). Signalons qu'il reproduit également la terminologie employée par Scoresby pour classer les différents types de cristaux de neige. Il s'intéresse également à la classification des cristaux de Moritz Anton Cappeller: là encore, il ne se contente pas de produire un recueil d'ornements disparates sur les cristaux. Ceci montre plus généralement l'intérêt de Bourgoin pour les classifications - qu'il s'agisse de cristaux ou de végétaux. Loin de se constituer un simple répertoire de formes, Bourgoin cherche à comprendre comment s'ordonnent les figures issues des planches scientifiques qu'il consulte. C'est pourquoi il reprend leur numérotation, pour les rapporter aux classifications correspondantes. Il semble donc séduit par l'esprit de système - souci d'exhaustivité et entreprise de classification cohérente - qui prédomine dans ses sources.

7. W. Scoresby, pl. IX du t. II de An Account of the Arctic Regions, 1820.

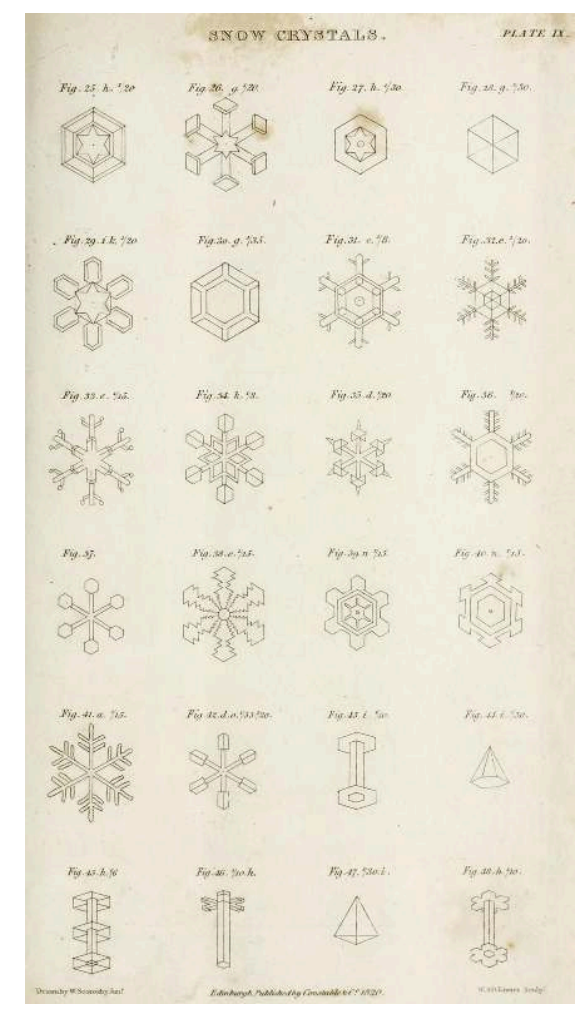

17 Au final, Bourgoin puise sa gamme de cristaux de neige dans six sources distinctes: Langwith, Musschenbroek, Stocke, Nettis, Scoresby et Schumacher. Ceci illustre son activité de recension exhaustive. Une fois cette collecte accomplie, il sélectionne par une croix rouge les cristaux de neige qui l'intéressent avant de les reproduire dans ses planches finales (fig. 8). Bourgoin a exclusivement recopié des figures issues de textes scientifiques. À cette transcription exacte s'ajoute le fait que les figures de la neige sont 
déclinées à partir de règles précises, édictées par les classifications scientifiques en s'en tenant à la déjà très grande variété des types de cristaux répertoriés par Nettis, Scoresby ou Schumacher.

\section{J. Bourgoin, Sélection d'étoiles de neige.}

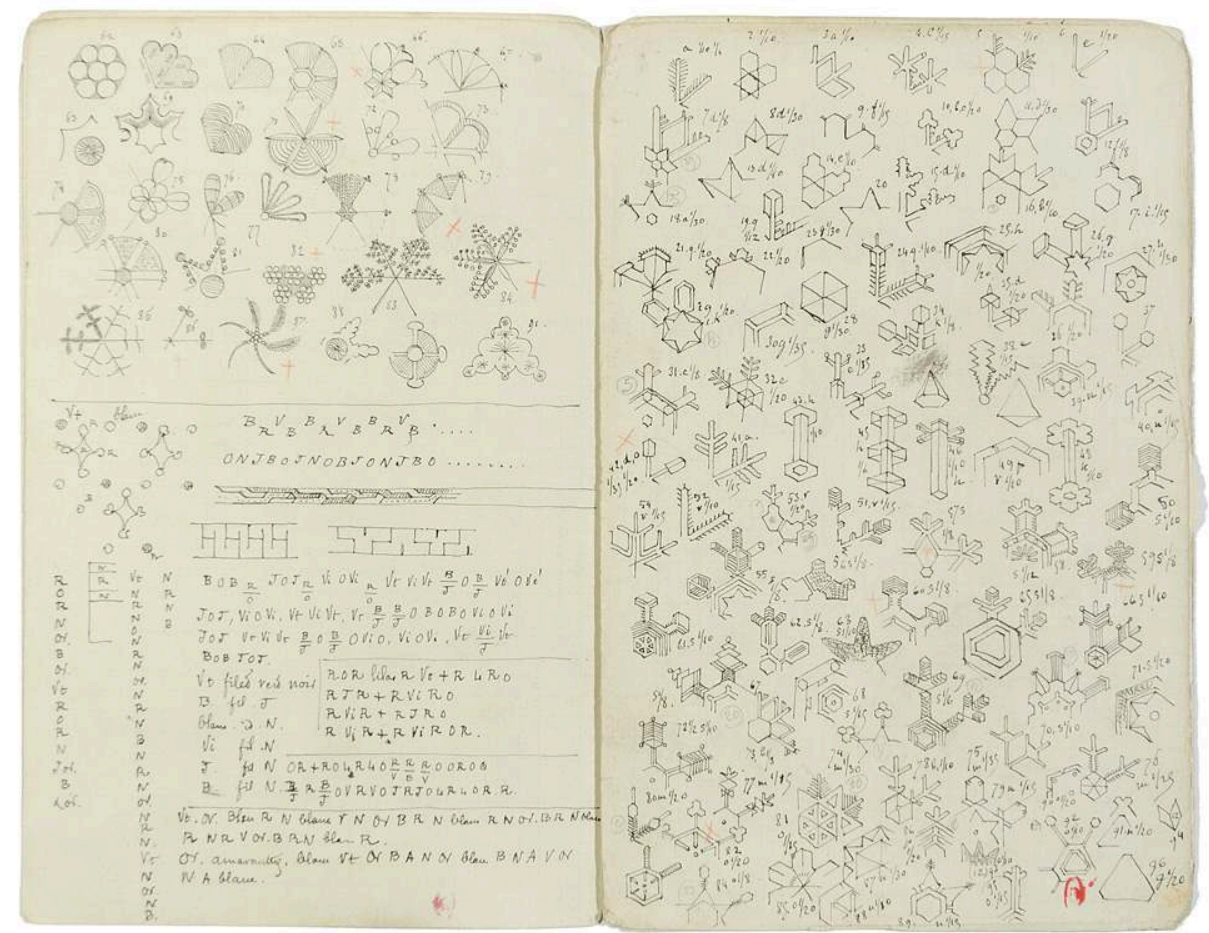

Source: Paris (France), Bibliothèque de l'Institut national d'histoire de l'art, collections Jacques Doucet, Arch. 67, 2, 1.

Les notes et croquis de Bourgoin sur les cristaux de neige forment ainsi un ensemble cohérent. Signalons toutefois que ces feuilles contiennent également des notes appartenant à des thématiques connexes, concernant les végétations métalliques ou des questions mathématiques ${ }^{21}$. Ajoutons que Bourgoin ne s'est pas non plus restreint aux cristaux de neige en consultant les Philosophical Transactions : par exemple, à côté du volume XXIV, Bourgoin a écrit « combinaisons ", qui semble renvoyer à un mémoire d'Edward Thornycroft ${ }^{22}$.

Parmi ces notes majoritairement consacrées aux cristaux se trouvent aussi plusieurs figures de Chladni, a priori sans parenté thématique, puisqu'il s'agit de dispositions régulières obtenues en faisant vibrer des plaques métalliques recouvertes de sable (fig. 9). En fait, Bourgoin a recopié un tableau issu d'un article de Charles Wheatstone ${ }^{23}$ publié dans un volume plus tardif des Philosophical Transactions, ce qui nous informe sur ses habitudes de lecture. S'il parcourt les périodiques scientifiques en privilégiant certaines entrées thématiques, il y trouve aussi d'autres planches intéressantes graphiquement. Bourgoin est d'autant plus sensible aux figures de Chladni qu'elles offrent un correspondant visuel aux vibrations acoustiques, ce qui rencontre son intérêt pour les correspondances entre phénomènes visuels et sonores, par exemple entre les couleurs et les gammes musicales. 


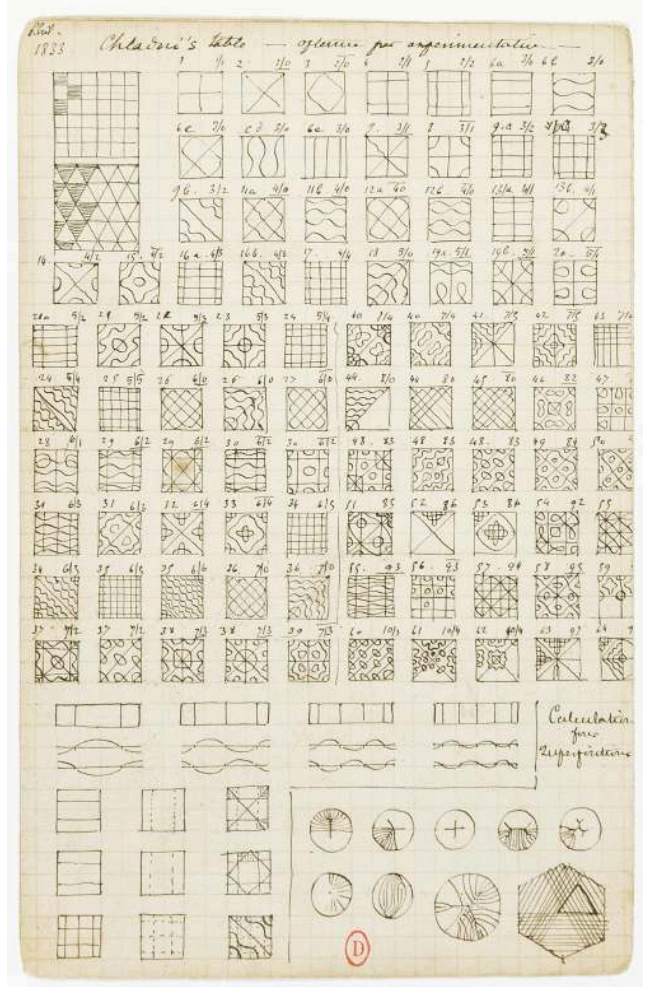

Source: Paris (France), Bibliothèque de l'Institut national d'histoire de l'art, collections Jacques Doucet, Arch. 67, 12, 3.

\section{Polygones et polyèdres : entre art ornemental, géométrie et cristallographie}

La thématique des polygones et des polyèdres se situe chez Bourgoin au croisement de la géométrie, de l'art ornemental et de la cristallographie. Il y revient au fil de ses écrits. Loin de s'intéresser à leurs seuls aspects formels et esthétiques, il prête attention à leurs propriétés mathématiques remarquables, élémentaires et plus complexes, comme en témoigne le large éventail de sources mobilisées sur ce thème. Les notes de Bourgoin indiquent des sources très variées, au-delà des écrits de Cournot et des nombreux manuels de géométrie élémentaire, dans lesquels plusieurs chapitres sont consacrés aux polygones, voire aux polyèdres. Loin de proposer une recension exhaustive de ces documents, nous nous restreindrons à quelques exemples susceptibles d'en éclairer les différents usages.

Dans ses ouvrages, Bourgoin distingue régulièrement plusieurs types de constructions : géométrique (à la règle et au compas), métrique (avec un décimètre par exemple) et empirique, cette dernière étant considérée par Bourgoin comme la plus spécifique aux artistes. Ces considérations se retrouvent également dans les chapitres dédiés aux polygones, par exemple dans Les Arts arabes:

Le problème de la division de la circonférence en parties égales ou de l'inscription des polygones réguliers dans le cercle, considéré au point de vue mathématique, dépend de la trigonométrie et se rattache à la théorie des équations algébriques. À ce point de vue très-général et purement spéculatif, c'est un fort beau problème, 
mais dont nous n'avons nul souci à prendre, car, aussi bien pour le géomètre que pour l'artisan, la division effective de la circonférence est une opération purement graphique et rentre dans les conditions d'exactitude que comportent toutes les opérations manuelles. ${ }^{24}$

Cette citation illustre bien sa volonté de préserver les arts des raisonnements mathématiques et d'approprier la géométrie aux pratiques de l'artisan. Loin de s'en tenir à des réflexions générales sur la géométrie, Bourgoin connaît et expose certaines constructions, exactes ou approchées, de polygones réguliers ${ }^{25}$, par ailleurs détaillées dans nombre de manuels de géométrie élémentaire. Sa culture mathématique gagne en profondeur lorsque paraît le deuxième volume des Études: il s'appuie alors sur des ouvrages mathématiques récents qui reviennent sur les questions de constructibilité à l'aide de certains instruments, comme par exemple la géométrie de la règle de Lambert ou le problème de la division du cercle en parties égales par Gauss ${ }^{26}$.

L'exemple d'un double feuillet sur lequel Bourgoin a recopié des extraits de textes en lien avec la construction des polygones réguliers, est particulièrement démonstratif ${ }^{27}$. Sur la troisième page, Bourgoin a reproduit in extenso un article d'Eugène-Charles Catalan sur le tétradécagone régulier ${ }^{28}$ (fig. 10,11,12). Sur le reste du double feuillet, il a sélectionné d'autres questions en lien avec le problème de Catalan. Ce dernier note que pour obtenir le côté du tétradécagone régulier, il est possible de s'appuyer sur la construction mise en œuvre dans le cas du décagone régulier. Or, le premier feuillet de Bourgoin renvoie à la proposition 10 du livre IV des Éléments d'Euclide, qui donne une construction d'un triangle isocèle dont les angles à la base sont les doubles de l'angle restant. Il s'agit là d'une étape fondamentale dans la construction du pentagone et du décagone réguliers. Le feuillet est complété par plusieurs figures illustrant cette proposition dans les cas du triangle et de l'hexagone, du carré et de l'octogone, du pentagone et du décagone. La deuxième page est consacrée à l'inscription de l'heptagone régulier dans un cercle, construction qui n'est pas possible à la règle et au compas car dépendant d'une équation du troisième degré. Enfin, au début de la quatrième page, Bourgoin note l'énoncé d'un problème classique sur le calcul du côté d'un polygone régulier à $2 n$ côtés à partir du côté d'un polygone régulier à $n$ côtés. À partir de ses notes "au propre ", Bourgoin travaille à nouveau sur ces questions au brouillon : il reproduit notamment la figure de Catalan, avec, au-dessus, une figure similaire pour le cas du décagone, et il les annote avec ses propres calculs. Ainsi, Bourgoin cherche activement à comprendre le texte de Catalan et à en restituer le raisonnement, en s'appuyant sur d'autres sources. 


\section{J. Bourgoin, Notes sur la construction du tétradécagone régulier d'après E. Catalan.}

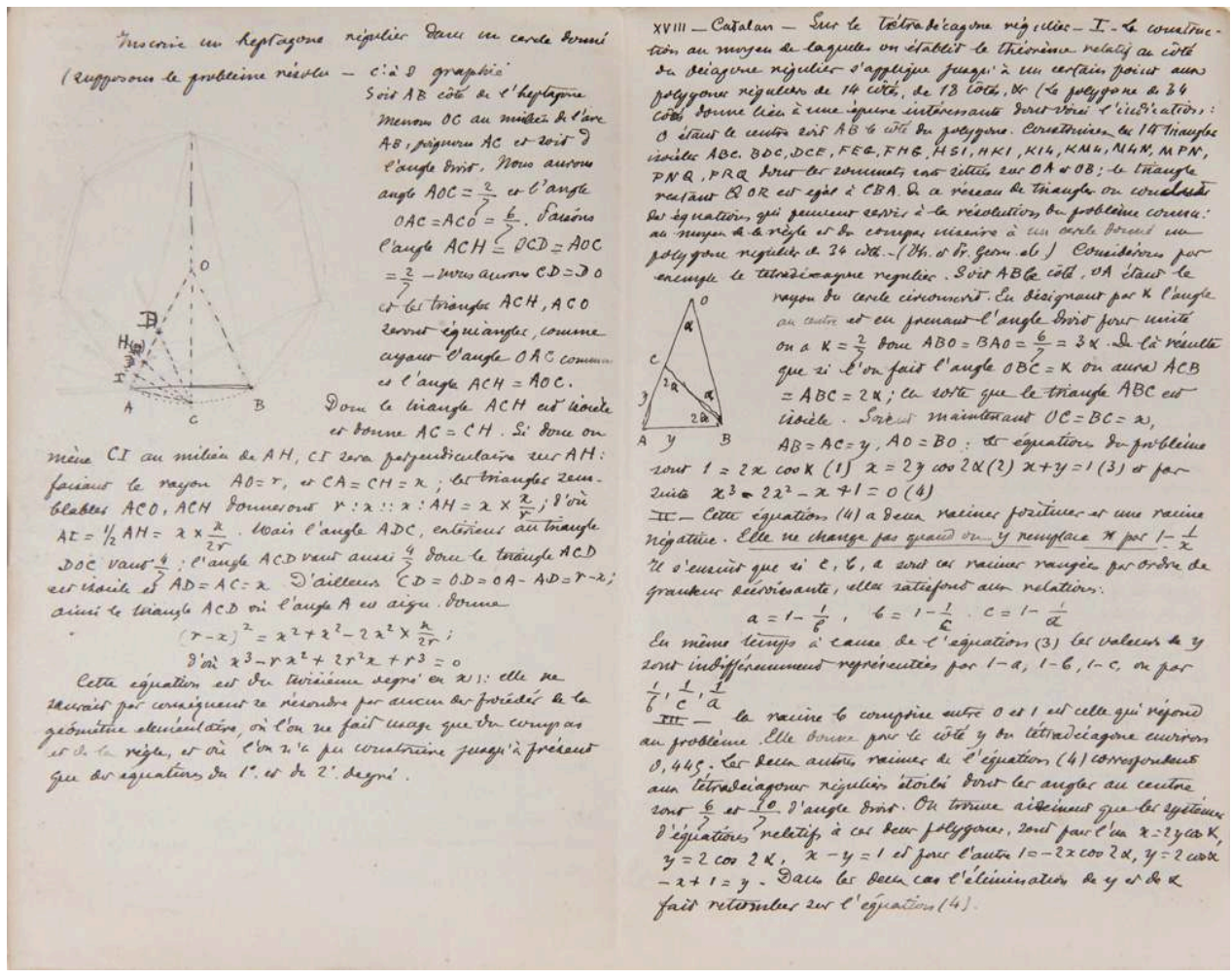

Source: Paris (France), Bibliothèque de l'Institut national d'histoire de l'art, collections Jacques Doucet, Arch. 67, 7, 2. 
11. J. Bourgoin, Notes sur la construction du tétradécagone régulier d'après E. Catalan.

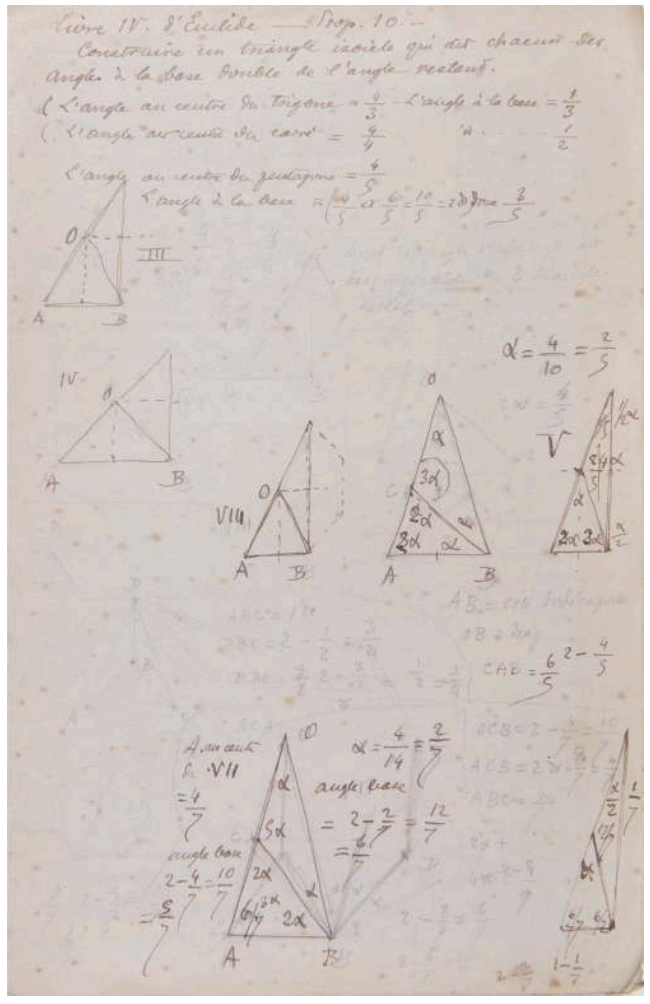

Source : Paris (France), Bibliothèque de l'Institut national d'histoire de l'art, collections Jacques Doucet, Arch. 67, 7, 2. 
12. J. Bourgoin, Notes sur la construction du tétradécagone régulier d'après E. Catalan.

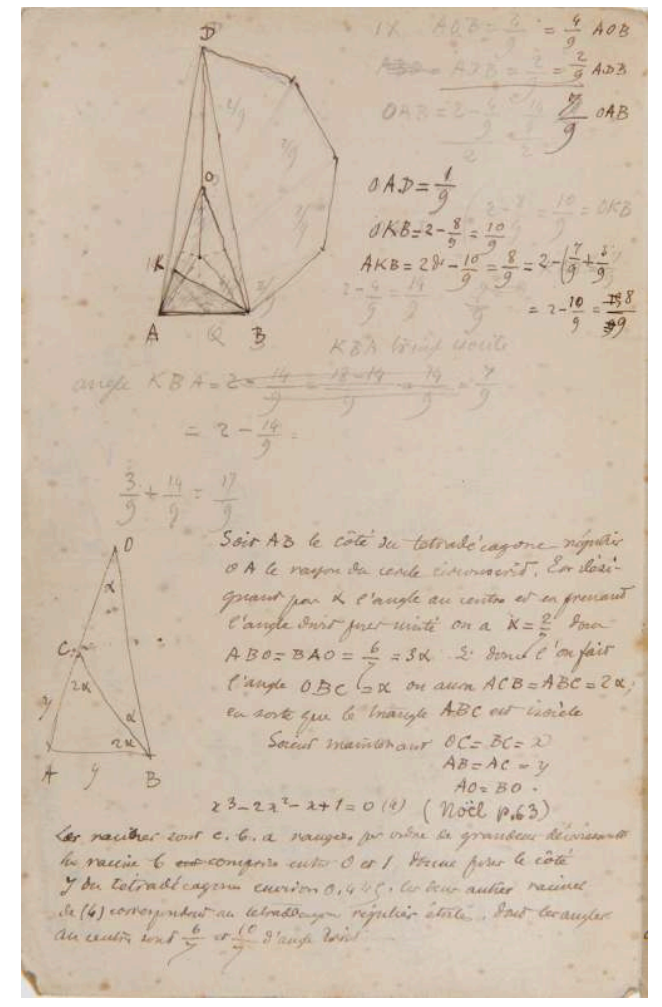

Source : Paris (France), Bibliothèque de l'Institut national d'histoire de l'art, collections Jacques Doucet, Arch. 67, 7, 2. Poinsot $^{29}$, auquel il a pu accéder par plusieurs biais. Il est avéré qu'il connaît les travaux de Poinsot via Cournot. De plus, ce mémoire est évoqué dans les nombreux manuels et articles traitant des polygones et polyèdres réguliers que Bourgoin a consultés. Enfin, ce dernier emprunte à l'éloge historique de Poinsot lu par Joseph Bertrand en 1890 un court passage en lien avec les polyèdres réguliers ${ }^{30}$.

Dans ses notes, Bourgoin ne relève aucun des raisonnements de Poinsot. En revanche, il sélectionne quelques extraits en vue d'alimenter certaines de ses thématiques de prédilection. Tout d'abord, il recopie une grande partie de l'introduction où Poinsot définit la géométrie de situation et où il met en avant l'importance des considérations d'ordre et de situation en mathématiques. Cela renvoie à l'intérêt de Bourgoin pour ces deux notions fondamentales. Il sélectionne plusieurs références convoquées par Poinsot à ce propos, par exemple Alexandre-Théophile Vandermonde sur lequel il revient dans le second volume des Études ${ }^{31}$.

Bourgoin reprend ensuite des résultats de Poinsot sur le nombre d'espèces de polygones réguliers, convexes et étoilés, que l'on peut former pour un « ordre » donné - l'ordre d'un polygone correspondant à son nombre de côtés - et sur certaines possibilités « d'espèces » données selon l'ordre du polygone - l'espèce étant définie par la somme de la mesure des angles. Bourgoin revient sur ces aspects dans plusieurs ouvrages $^{32}$ où il y répertorie les polygones étoilés existants pour un nombre de côtés donnés, en admettant toutefois des figures exclues par Poinsot. Ainsi, bien qu'il n'existe pas de polygone étoilé au sens de Poinsot dans le cas de l'hexagone, Bourgoin comptabilise, lui, la figure composée de deux triangles équilatéraux entrecroisés. 

des polyèdres. Celle-ci fait l'objet de plusieurs mémoires à la fin des années 1850 et d'une question pour le Grand prix de mathématiques de l'Académie des sciences en 1861 et en 1863. Bourgoin s'est justement constitué à ce sujet un corpus important de sources publiées entre 1858 et 1865, auxquelles il a peut-être eu accès par l'intermédiaire de manuels de géométrie élémentaire. Par exemple, les Théorèmes et problèmes de géométrie élémentaire (1865) de Catalan, auxquels renvoient plusieurs notes de Bourgoin, s'achèvent sur un appendice consacré aux polyèdres semi-réguliers. Catalan y annonce un mémoire à paraitre dans le Journal de l'École polytechnique, qui est issu d'un travail présenté à l'Académie dans le cadre du concours de 1863. Bourgoin prend non seulement des notes sur ce mémoire ${ }^{34}$ mais remonte également les filières bibliographiques à partir de références listées par Catalan, dont Poinsot puis Lidonne ${ }^{35}$. Il semble même que Bourgoin ait feuilleté les Comptes rendus de l'Académie des sciences de l'année 1863, à partir de la remarque de Catalan sur l'objet du Grand prix de

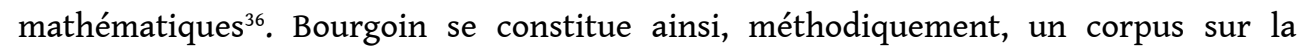
thématique des polyèdres.

Là encore, il opère des choix dans sa lecture de Catalan. Après avoir recopié sa définition des polyèdres semi-réguliers, il sélectionne quelques théorèmes généraux sur les polyèdres, puis il synthétise sa classification des polyèdres semi-réguliers. En revanche, il ne prend aucune note sur la méthode de Catalan pour obtenir la liste des différents solides. Ces feuillets sont suivis d'autres notes de Bourgoin, issues cette foisci du texte de Lidonne: comme précédemment, Bourgoin emprunte à ce dernier sa classification des solides d'Archimède tout en relevant les nouvelles références données. Dans les deux cas, Bourgoin reproduit également une partie des figures insérées à la fin des volumes consultés. Les solides de Catalan réapparaissent également dans d'autres carnets ${ }^{37}$ (fig. 13). 
13. J. Bourgoin, Polyèdres.

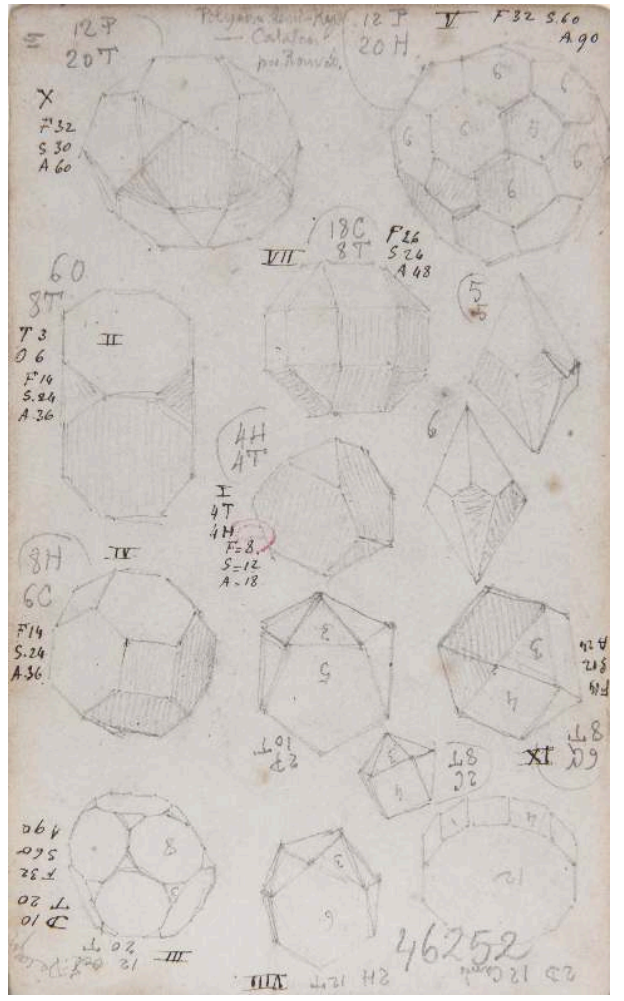

Source : Paris (France), Bibliothèque de l'Institut national d'histoire de l'art, collections Jacques Doucet, Arch. 67, 9, 22.

Signalons enfin que Bourgoin relève systématiquement les quelques passages dans lesquels Lidonne et Catalan relient les polyèdres à la cristallographie. Ce lien intéresse Bourgoin dès la publication de ses premiers ouvrages. Il y revient également en fin de carrière, puisqu'il consulte alors des travaux plus spécialisés en cristallographie ${ }^{38}$.

L'ensemble des notes de Bourgoin offre un panorama très riche de ses sources scientifiques. Il est possible de mettre en évidence des thématiques transversales, qui impliquent de ne pas séparer les domaines scientifiques investis par Bourgoin: par exemple son intérêt pour les classifications et pour la morphologie se manifeste en botanique, pour les cristaux et les polyèdres. Il apparaît à quel point Bourgoin est méthodique dans la constitution de corpus thématiques, malgré le désordre apparent de certaines liasses de notes. Selon les cas, il s'appuie sur une ou plusieurs « sources mères » et il relève les références données par les auteurs en vue d'une consultation ultérieure.

Dans le cas plus spécifique des mathématiques, il est en mesure de faire par lui-même le lien entre diverses sources; par exemple à propos du tétradécagone. Les notes de Bourgoin témoignent d'ailleurs d'une pratique effective et régulière des mathématiques, en particulier au moment de l'élaboration de ses Études architectoniques, où l'auteur poursuit un dialogue critique avec les manuels de géométrie élémentaire ${ }^{39}$. Ainsi, les sciences mathématiques, qui occupent une place privilégiée dans le corpus de Bourgoin, sont mobilisées à différents effets: pour appréhender certaines figures élémentaires - polygones et polyèdres -, pour préciser les contours de sa graphique et pour repenser les liens entre géométrie, dessin, industrie et art ornemental. 


\section{ANNEXES}

Panorama non exhaustif des sources scientifiques consultées par Bourgoin.

Cette liste a été établie d'après des notes de lecture conservées à la Bibliothèque de l'Institut national d'histoire de l'art (Arch. 67) et à la médiathèque de Joigny (archives 64).

\section{Ouvrages de Cournot}

COURNOT Antoine-Augustin, Exposition de la théorie des chances et des probabilités, Paris : Hachette, 1843.

COURNOT Antoine-Augustin, De l'origine et des limites de la correspondance entre l'algèbre et la géométrie, Paris : Hachette, 1847.

COURNOT Antoine-Augustin, Essai sur les fondements de nos connaissances et sur les caractères de la critique philosophique, Paris : Hachette, 1851.

COURNOT Antoine-Augustin, Traité de l'enchaînement des idées fondamentales dans les sciences et dans l'histoire, Paris : Hachette, 1861.

COURNOT Antoine-Augustin, Considérations sur la marche des idées et des événements dans les Temps modernes, Paris : Hachette, 1872.

COURNOT Antoine-Augustin, Matérialisme, vitalisme, rationalisme. Études sur l'emploi des données de la science en philosophie, Paris : Hachette, 1875.

\section{Manuels de mathématiques}

BOBILLIER Étienne E., Cours de géométrie, Paris : Hachette, 1880.

CAMus Charles-Étienne-Louis, Cours de mathématiques. Seconde partie. Élémens de géométrie théorique et pratique, $4^{e}$ éd., Paris : P. E. G. Durand, 1769.

CATALAN Eugène-Charles, Théorèmes et problèmes de géométrie élémentaire, $4^{\mathrm{e}}$ éd., Paris : Dunod, 1865.

COMBEROUSSE Charles de, Cours de mathématiques, à l'usage des candidats à l'école polytechnique : t. deuxième : Géométrie élémentaire, plane et dans l'espace. Trigonométrie rectiligne et sphérique, Paris : Gauthier-Villars, 1882.

COUSINERY Barthélémy Édouard, Géométrie perspective, ou Principes de projection polaire appliqués à la description des corps, Paris : Carilian-Goeury, 1828.

HACHETTE Jean Nicolas P., Éléments de géométrie à trois dimensions, Paris : Courcier, 1817.

LA GOURNERIE Jules de, Traité de géométrie descriptive, Paris : Gauthier-Villars, 1873.

LEGENDRE Adrien-Marie, Éléments de géométrie, avec des notes, $12^{\mathrm{e}}$ éd., Paris : Firmin Didot, 1823.

MAZÉAS Jean-Mathurin, Elémens d'arithmétique, d'algèbre et de géométrie, $3^{\mathrm{e}}$ éd., Paris : Le Mercier, 1765. 
ROUCHÉ Eugène, Éléments de statique graphique : polygones et courbes funiculaires, ligne élastique, centres de gravité et moments d'inertie, poutre droite sur deux appuis, forces intérieures dans les systèmes articulés, poutre continue, Paris : Baudry et C $C^{\text {ie }}, 1889$.

ROUCHÉ Eugène et COMBEROUSSE Charles de, Traité de géométrie élémentaire, Paris : Gauthier-Villars, 1866.

ROUCHÉ Eugène et COMBEROUSSE Charles de, Traité de géométrie, Paris : Gauthier-Villars, 1891.

ROUCHÉ Eugène et COMBEROUSSE Charles de, Leçons de géométrie rédigées suivant les derniers programmes officiels, Paris : Gauthier-Villars, 1896.

SAVÉRIEN Alexandre, Dictionnaire universel de mathématiques et de physique, Paris : J. Rollin, 1753.

\section{Ouvrages et articles divers en mathématiques}

CASTEL Louis-Bertrand, Mathématique universelle abrégée, à l'usage et à la portée de tout le monde, Paris : Pierre Simon, 1728.

CHASLES Michel, Aperçu historique sur l'origine et le développement des méthodes en géométrie particulièrement de celles qui se rapportent à la géométrie moderne, Bruxelles : Hayez, 1837.

CONDAMINE Charles Marie de La, «Recherches sur le tour ", Mémoires de l'Académie royale des sciences, 1734, p. 216-258, 295-340.

HOEFER Ferdinand, Histoire des mathématiques : depuis leurs origines jusqu'au commencement du dix-neuvième siècle, Paris : Hachette, 1874.

KLEIN Félix, Leçons sur certaines questions de géométrie élémentaire, Paris : Librairie Nony, 1896.

моUснот Augustin-Bernard, La Réforme cartésienne : étendue aux diverses branches des mathématiques pures, Paris : Gauthier-Villars, 1876.

SARRAZIN DE MONTFERRIER Alexandre (éd.), Dictionnaire des sciences mathématiques pures et appliquées, Bruxelles : A. de Mat, 1838.

VANDERMONDE Alexandre-Théophile, « Remarques sur les problèmes de situation », Histoire de l'Académie royale des sciences, avec les Mémoires de mathématiques et de physique, Année 1771, 1774, p. 566-574.

\section{Combinatoire}

LAMBERT Johann Heinrich, « Essai de taxéométrie, ou sur la mesure de l'ordre », Nouveaux mémoires de l'Académie royale des sciences et belles-lettres de Berlin, Année 1770, 1772 , p. 327-342.

LAMBERT Johann Heinrich, "Second essai de taxéométrie, ou sur la mesure de l'ordre », Nouveaux mémoires de l'Académie royale des sciences et belles-lettres de Berlin, Année 1773, 1775 , p. 347-368.

THORNYCROFT Edward, « The Doctrine of Combinations and alternations », Philosophical Transactions, vol. 24, 1705-1704, p. 1961-1970.

Polygones et polyèdres 
ANONYME, « Des nouveaux cors réguliers qui se peuvent faire avec les primitifs », Journal des savants, 1698, p. 183-187.

BERTRAND Joseph, « Note sur la théorie des polyèdres réguliers », Comptes rendus hebdomadaires des séances de l'Académie des sciences, vol. 46, 1858, p. 79-82.

BERTRAND Joseph, Éloge historique de Louis Poinsot, Paris : Institut de France, 1890.

BRETON Ph., « Note sur la classification des polyèdres ", Comptes rendus hebdomadaires des séances de l'Académie des sciences, vol. 51, 1860, p. 722-724.

CATALAN Eugène-Charles, « Mémoire sur la théorie des polyèdres ", Journal de l'École polytechnique, vol. 24, 41 eahier, 1865, p. 171.

CATALAN Eugène-Charles, « Sur le tétradécagone régulier », dans Mélanges mathématiques, Liège : F. Hayez, 1885, p. 1-71.

CAUCHY Augustin Louis, " Recherches sur les polyèdres : premier mémoire ", Journal de l'École polytechnique, vol. 9, 16 eahier, 1813, p. 68-86.

CAUCHY Augustin Louis, "Sur les polygones et les polyèdres : second mémoire ", Journal de l'École polytechnique, vol. 9, 16e cahier, 1813, p. 87-98.

GERGONNE Joseph-Diez, « Géométrie élémentaire. Recherche de quelques-unes des lois générales qui régissent les polyèdres ", Annales de mathématiques pures et appliquées, vol. 15, 1824-1825, p. 157-164.

LIDONNE Nicolas-Joseph, Tables de tous les diviseurs des nombres, calculées depuis un jusqu'à cent deux mille, Paris : Courcier, 1808.

POINSOT Louis, « Mémoire sur les polygones et les polyèdres ", Journal de l'École polytechnique, vol. 4, 10 cahier, 1810, p. 16-49.

POINSOT Louis, " Note sur la théorie des polyèdres ", Comptes rendus hebdomadaires des séances de l'Académie des sciences, vol. 46, 1858, p. 65-79.

\section{Mécanique}

DELAUNAy Charles, Cours élémentaire de mécanique théorique et appliquée, Paris : V. Masson, 1851.

HATON DE LA GOUPILLIÈRE Julien Napoléon, « La géométrie des masses », Revue générale des sciences pures et appliquées, vol. 4, 1893.

LABOUlaye Charles Pierre Lefebvre de, Traité de cinématique théorique et pratique, Paris : L. Mathias, 1849-1854.

\section{Botanique}

BRAVAIS Auguste et BRAVAIS Louis, « Essai sur la disposition des feuilles curvisériées ", Annales des sciences naturelles (Botanique), vol. 7, 1837, p. 42-110.

BRAVAIS Auguste et BRAVAIS Louis, « Essai sur la disposition symétrique des inflorescences ", Annales des sciences naturelles (Botanique), vol. 8, 1838, p. 11-42. BRAVAIS Auguste et BRAVAIS Louis, « Essai sur la disposition générale des feuilles rectisériées ", Annales des sciences naturelles (Botanique), vol. 12, 1839, p. 5-51, 65-77. 
CANDOLLE Alphonse de, Phytographie ou L'art de décrire les végétaux considérés sous différents points de vue, Paris : G. Masson, 1880.

FERMOND Charles, Études sur la symétrie : considérée dans les trois règnes de la nature, Paris : Chaix et Cie, 1855.

FERMOND Charles, Essai de phytomorphie, tome I, Paris : Germer Baillère, 1864.

FERMOND Charles, Phytogénie, ou théorie mécanique de la végétation, Paris : Germer Baillère, 1867.

Cristallographie

CAPPELLER Anton Moritz, Prodromus crystallographia de crystallis improprie sic dictis, Lucerne : Typis Henrici Rennwardi Wyssing, 1723.

HAÜY René-Just, Traité de cristallographie, Paris : Bachelier et Huzard, 1822.

ROMÉ DE L'ISLE Jean Baptiste Louis de, Cristallographie, ou description des formes propres à tous les corps du règne minéral, Paris : Imprimerie de Monsieur, 1783.

SORET Charles, Éléments de cristallographie physique, Genève et Paris : Georg et GauthierVillars, 1893.

\section{Cristaux de neige}

BRAVAIS Auguste, «Sur l'indice de réfraction et la dispersion de la glace », Annales de chimie et de physique, vol. 21, 1847, p. 361-363.

BRAVAIS Auguste, « Sur les halos et les phénomènes optiques qui les accompagnent ", Journal de l'école polytechnique, vol. 18, 31 eahier, 1847, p. 1-270.

GREW Nehemia, «Some Observations Touching the Nature of Snow », Philosophical Transactions, vol. 8, 1673, p. 5193-5196.

HOOKE Robert, Micrographia, Londres : Royal Society of London, 1667.

LANGWITH Benjamin, « Observations on the Figure of Snow », Philosophical Transactions, vol. 32, 1722, p. 298-300.

MUSSCHENBRoeK Petrus van, «Ephemerides Meteorologicae », Philosophical Transactions, vol. 37, 1731-1732, p. 357-384.

MUSSCHENBRoeK Petrus van, Elements of Natural Philosophy, Londres : J. Nourse, 1744. MUSSCHENBROEK Petrus van, Introductio ad philosophiam naturalem, Lugduni Batavorum : Apud S. et J. Luchtmans, 1762.

NETTIS Johann, « An Account of a Method of Observing the Wonderful Configurations of the Smallest Shining Particles of Snow », Philosophical Transactions, vol. 49, 1756, p. 644-648.

SCHUMACHER Georg Friedrich, Die Krystallisation des Eises, Leipzig : Mayer und Wigand, 1844.

SCORESBY William, An Account of the Artic Regions, Edinburg : A. Constable \& Company, 1820. 
STOCKE Leonard, « Enarratio observationum circa Rorem deciduum... », Philosophical Transactions, vol. 42, 1742-1743, p. 112-114.

VENEL Gabriel François, « Article «neige» », dans Encyclopédie ou dictionnaire raisonné des sciences, des arts et des métiers, Neufchastel : Samuel Faulche et C $C^{\text {ie }}, 1765$, p. 88.

WILCKE Johan Carl, « Rön och tankar om snö-figurers skiljaktighet [expérience et réflexion sur la variété des figures de la neige], ", Kongliga Vetenskapsacademiens Handlingar [Mémoires de l'Académie royale des sciences de Stockholm], 1761, p. 120.

Stéréochimie

HANTZSCH Arthur Rudolf, Précis de stéréochimie, Paris : G. Carré, 1896.

MONOD Édouard-Gabriel, Stéréochimie, exposé des théories de Le Bel et Van't Hoff, Paris : Gauthier-Villars, 1895.

Couleurs

CHEVREUL Michel-Eugène, « Mémoire sur l'influence que deux couleurs peuvent avoir l'une sur l'autre quand on les voit simultanément ", Mémoires de l'Académie royale des sciences de l'Institut de France, vol. 11, 1832, p. 447-520.

CHEVREUL Michel-Eugène, De la loi du contraste simultané des couleurs : et de l'assortiment des objets colorés, considéré d'après cette loi, Paris : Pitois-Levrault et Cie, 1839.

CHEVREUL Michel-Eugène, « Note sur quelques expériences de contraste simultané des couleurs ", Comptes rendus hebdomadaires des séances de l'Académie des sciences, vol. 47, 1858, p. 196-198.

CHEVREUL Michel-Eugène, « Exposé d'un moyen de définir et de nommer les couleurs », Mémoires de l'Académie des sciences de l'Institut de France, vol. 33, 1861, p. 3-944.

CUREAU DE LA CHAMBRE Marin, Nouvelles observations sur l'iris, Paris : Jacques d'Allin, 1662.

DOLLFUS-AUSSET Daniel, Matériaux pour la coloration des étoffes, Paris : F. Savy, 1865.

FAIVRE Ernest, CEuvres scientifiques de Goethe, Paris : L. Hachette, 1862.

MAIRAN Jean-Jacques Dortous de, « Troisième partie des recherches physicomathématiques sur la réflexion des corps ", Mémoires de l'Académie royale des sciences de l'Institut de France, 1738, p. 1-65.

MÉRIMÉE Jean-François Léonor, De la peinture à l'huile ou, des procédés matériels employés dans ce genre de peinture, depuis Hubert et Jean Van-Eyck jusqu'à nos jours, Paris : Huzard, 1830.

PLATEAU Joseph, «Sur le phénomène des couleurs accidentelles », Annales de chimie et de physique, vol. 53, 1833, p. 396-398.

SZOKALSKI Victor, Essai sur les sensations des couleurs dans l'état physiologique et pathologique de l'œil, Paris : H. Cousin, 1840.

Végétations métalliques

CONDAMINE Charles Marie de La, «Sur une nouvelle espèce de végétation métallique », Mémoires de l'Académie royale des sciences, 1730, p. 466-482. 
HOMBERG Wilhelm, « Mémoire touchant les végétations artificielles », Mémoires de l'Académie royale des sciences, 1710, p. 426-438.

PARROT Georg Friedrich von, « Essai sur le procès de la végétation métallique et de la cristallisation ", Mémoires de l'Académie impériale des sciences de Saint-Pétersbourg Sciences mathématiques et physiques, II (6e série), 1841, p. 493-674.

\section{NOTES}

1. Voir Jenny BOUCARD et Christian ECKES, «Théorie de l'ordre et syntactique chez Jules Bourgoin ", infra, p. 281-299.

2. Des notes de lecture sont conservées dans le fonds d'archives Bourgoin de la Bibliothèque de l'Institut d'histoire de l'art, collections Jacques Doucet, Arch. 67 et à la médiathèque de Joigny, Yonne (notamment 64.14). En annexe figure une liste des principaux périodiques et ouvrages cités ou consultés par Bourgoin sur les thèmes évoqués.

3. Paris (France), Bibliothèque de l'Institut national d'histoire de l'art, collections Jacques Doucet, Arch. 67, 1, 1 .

4. Antoine-Augustin counnot, De l'origine et des limites de la correspondance entre l'algèbre et la géométrie, Paris : Hachette, 1847.

5. Le fonds Isidore Francœur de la bibliothèque de l'École nationale supérieure des beaux-arts est particulièrement riche en traités de mathématiques.

6. Cet inventaire établi du 23 au 25 mai 1908 nous a été communiqué sous forme de photocopie.

7. Il consulte par exemple les différentes publications de l'Académie des sciences de Paris, les Mémoires de l'Académie de Berlin, les Philosophical Transactions, les Mémoires de l'Académie de Saint-Pétersbourg, les Annales de Chimie et de Physique, les Annales des sciences naturelles, la Revue générale des sciences pures et appliquées, la Revue philosophique de la France et de l'étranger, le Journal de l'École polytechnique et, de manière plus sporadique, les Annales de mathématiques pures et appliquées ainsi que les Nouvelles annales de mathématiques.

8. Auguste BRAVAIS et Louis BRAVAIS, "Essai sur la disposition générale des feuilles rectisériées ", Annales des sciences naturelles (Botanique), vol. 12, 1839, p. 5-51, 65-77; Charles FERMOND, Essai de phytomorphie, tome 1, Paris : Germer Baillière, 1864.

9. Jules BouRgoin, Théorie de l'ornement, Paris: A. Lévy, 1873, p.110-111, 297 ; ID., Grammaire élémentaire de l'ornement, Paris : Ch. Delagrave, 1880, p. 123-140.

10. Paris (France), Bibliothèque de l'Institut d'histoire de l'art, collections Jacques Doucet, Arch. $67,8$.

11. Paris (France), Bibliothèque de l'Institut d'histoire de l'art, collections Jacques Doucet, Arch. $67,2,2$ (pour les croquis préparatoires) et 67,15 (pour les planches).

12. Voir Estelle thiBault, «Le projet d'une "Revue de l'ornement" (1877)», in Jules Bourgoin 1838-1908. L'obsession du trait (catalogue de l'exposition Paris, Institut national d'histoire de l'art, 20 novembre 2012-12 janvier 2013), Paris, INHA, 2012, p. 20.

13. Georg Friedrich schumacher, Die Krystallisation des Eises, Leipzig : Mayer und Wigand, 1844.

14. Robert HоOKE, Micrographia, London : Royal Society of London, 1667.

15. Petrus van MUSSChEnBroek, «Ephemerides Meteorologicae », Philosophical Transactions, vol. 37, 1731-1732, p. 357-384. Bourgoin reproduit les figures 1 à 4 du Tableau 2.

16. C'est notamment le cas de: Gabriel François VEnEL, "Article "neige" ", dans Encyclopédie ou dictionnaire raisonné des sciences, des arts et des métiers, Neufchastel : Samuel Faulche et Cie, 1765, p. 88. 
17. Benjamin LANGWITH, « Observations on the Figure of Snow », Philosophical Transactions, vol. 32, 1722, p. 298-300. Bourgoin a notamment recopié la figure 3.

18. Johann NETTIS, "An Account of a Method of Observing the Wonderful Configurations of the Smallest Shining Particles of Snow », Philosophical Transactions, vol. 49, 1756, p. 644-648.

19. L'orthographe «Wilke », sans le « $\mathrm{c} »$, provient de l'ouvrage de Schumacher.

20. William SCORESBY, An Account of the Arctic Regions, Edinburgh : A. Constable \& Company, 1820, t. 1 et 2. Les planches VIII à XI du second volume contiennent 96 figures de cristaux de neige que Bourgoin condense sur une seule feuille, en respectant la numérotation.

21. Christophe-Bernard DE BRAGELONGNE, «Examen des lignes du quatrième ordre, ou courbe du troisième genre ", Mémoires de l'Académie royale des sciences, 1730, p. 158-216 ; Nicolas Fuss, « De la division d'un rhomboïde en quatre parties égales, par deux lignes droites qui se coupent à angles droits ", Mémoires de l'Académie impériale des sciences de Saint-Pétersbourg, tome 3, 1809-1810, p. 65-74; Georg Friedrich VON PARROT, «Essai sur le procès de la végétation métallique et de la cristallisation", Mémoires de l'Académie impériale des sciences de Saint-Pétersbourg - Sciences mathématiques et physiques, tome 2 ( $6^{\mathrm{e}}$ série), 1841, p. 493-674.

22. Edward THORNYCROFT, "The Doctrine of Combinations and Alternations ", Philosophical Transactions, vol. 24, 1705-1704, p. 1961-1970.

23. Charles WHEATSTONE, "On the Figures Obtained by Strewing Sand on Vibrating Surfaces, Commonly Called Acoustic Figures », Philosophical Transactions, vol. 123, 1833, p. 593-633.

24. Jules Bourgoin, Les Arts arabes, Paris : V ve A. Morel, 1873, p. 8.

25. Ibid., p. 7-9.

26. Bourgoin reproduit ainsi de longs extraits de Franz WOEPCKE, « Analyse et extrait d'un recueil de constructions géométriques, par Aboûl-Wafâ », Journal asiatique, tome 5 ( $5^{\mathrm{e}}$ série), 1855 ainsi que de Félix KLEIN, Leçons sur certaines questions de géométrie élémentaire, Paris : Librairie Nony, 1896.

27. Paris (France), Bibliothèque de l'Institut national d'histoire de l'art, collections Jacques Doucet, Arch. 67, 7, 2.

28. Eugène-Charles CATALAN, «Sur le tétradécagone régulier », in Mélanges mathématiques, Liège :

F. Hayez, 1885.

29. Louis POINSOT, «Mémoire sur les polygones et les polyèdres ", Journal de l'École polytechnique, vol. 4 (X cahier), 1810, p. 16-49. Voir les notes de Bourgoin, INHA, Arch. 67, 11, 3.

30. Joseph BERTRAND, Éloge historique de Louis Poinsot, Paris : Institut de France, 1890, p. LXXXV.

31. Jules BouRgoIN, Études architectoniques et graphiques, t. 2, Paris : Ch. Schmid, 1901, p. 20.

32. Par exemple : Jules BouRgoIn, Théorie..., op. cit. (note 9), p.143-147; ID., Les Arts arabes, op.cit. (note 24), p. 10-11.

33. Nicolas-Joseph LIDONNE, Tables de tous les diviseurs des nombres, calculées depuis un jusqu'à cent deux mille, Paris : Courcier, 1808.

34. Eugène-Charles CATALAN, "Mémoire sur la théorie des polyèdres", Journal de l'École polytechnique, vol. 24, XLI ${ }^{\mathrm{e}}$ cahier, 1865, p. 1-71. Voir les notes de Bourgoin, INHA, Arch. 67, 3, 1.

35. C'est notamment le cas de: Louis PoINSOT, "Note sur la théorie des polyèdres ", Comptes rendus hebdomadaires des séances de l'Académie des sciences, vol.46, 1858, p.65-79; Ph. BRETON, "Note sur la classification des polyèdres ", Comptes rendus hebdomadaires des séances de l'Académie des sciences, vol. 51, 1860, p. 722-724.

36. Sur un des feuillets contenant les notes de Bourgoin sur les Comptes rendus de l'Académie des sciences de 1860, on trouve l'indication « Revoir C. R. 1863 ».

37. Paris (France), Bibliothèque de l'Institut national d'histoire de l'art, collections Jacques Doucet, Arch. 67, 9, carnets 22 et 23. 
38. Voir Jules Bourgoin, Théorie..., op. cit. (note 9), p. 276-277. Dans des notes conservées à la bibliothèque de Joigny (64.14), Bourgoin a par exemple recopié le début de Charles SORET, Éléments de cristallographie physique, Genève et Paris : Georg et Gauthier-Villars, 1893.

39. Voir Estelle thiBAULt, « La graphique, une science des figures pour l'école et l'atelier », supra, p. $255-280$.

\section{AUTEURS}

\section{JENNY BOUCARD}

Jenny Boucard est maître de conférences en histoire des sciences à l'université de Nantes et membre du centre François-Viète. Ses travaux portent sur l'histoire de la théorie des nombres (second XVIII ${ }^{\mathrm{e}}$ siècle-XIX ${ }^{\mathrm{e}}$ siècle) et sur la théorie de l'ordre.

\section{CHRISTOPHE ECKES}

Christophe Eckes est maître de conférences en mathématiques, spécialisé en histoire des mathématiques, à l'université de Lorraine et membres des Archives Henri Poincaré. Ses travaux portent sur l'histoire de l'œuvre mathématique et philosophique de Hermann Weyl (1885-1955), l'histoire de la perspective aux XVIII et XIX ${ }^{\mathrm{e}}$ siècles et sur l'histoire de l'art ornemental au XIX siècle. 